OPEN ACCESS

Edited by:

Rui Cao,

Capital Medical University, China

Reviewed by:

Peixin Dong

Hokkaido University, Japan

Fukang Sun

Shanghai Jiao Tong University, China

*Correspondence: Kaixing $A$

wszk215@163.com; akxing8258@tongji.edu.cn

Specialty section: This article was submitted to Protein and RNA Networks, a section of the journal Frontiers in Molecular Biosciences

Received: 24 March 2021 Accepted: 12 April 2021 Published: 13 May 2021

Citation:

Zhang R, Zhu W, Ma C and Ai K (2021) Silencing of circRNA circ_0001666 Represses EMT in Pancreatic Cancer Through Upregulating miR-1251 and Downregulating SOX4.

Front. Mol. Biosci. 8:684866. doi: 10.3389/fmolb.2021.684866

\section{Silencing of circRNA circ_0001666 Represses EMT in Pancreatic Cancer Through Upregulating miR-1251 and Downregulating SOX4}

\author{
Rundong Zhang ${ }^{1}$, Wanli Zhu ${ }^{1}$, Chenchao $\mathrm{Ma}^{2}$ and Kaixing $\mathrm{Ai}^{{ }^{*}}{ }^{*}$ \\ ${ }^{1}$ Department of General Surgery, Shanghai Pulmonary Hospital, Tongji University School of Medicine, Shanghai, China, \\ ${ }^{2}$ Department of Thoracic Surgery, Shanghai Pulmonary Hospital, Tongji University School of Medicine, Shanghai, China
}

Background: Pancreatic cancer (PC) is an aggressive malignancy and has a poor prognosis. Although emerging research has revealed that circular RNAs (circRNAs) are crucial modulators that control tumor development and metastasis, their functional involvement in PC has not been well characterized. Here, we examined whether and how circRNA circ_0001666 governs epithelial-mesenchymal transition (EMT) in PC.

Methods: We investigated the effects of circ_0001666 on EMT and PC cell invasion by gain- and loss-of-function assays. We also explored the mechanisms underlying the functions of circ_0001666 in PC cells.

Results: We found that circ_0001666 is highly expressed in PC tissues and PC cell lines. Patients with high circ_0001666 expression had shorter survival times. In vitro and in vivo experiments have demonstrated that upregulation of circ_0001666 facilitates PC cell proliferation, EMT and invasiveness, whereas knockdown of circ_0001666 exhibits opposite functions. Moreover, circ_0001666 is able to bind to miR-1251, thus increasing the expression of SOX4, which is a direct downstream effector of miR-1251. The oncogenic effects of circ_0001666 on EMT and PC cell invasion were rescued by miR-1251 overexpression.

Conclusions: These results suggested that circ_0001666 acts as an oncogenic circRNA to promote EMT and invasion of PC cells through sponging miR-1251, and indicated that circ_0001666 could be explored as a potential therapeutic target for PC.

Keywords: circ_0001666, circular RNA, circRNA, pancreatic cancer, microRNA-1251, EMT, SOX4

\section{BACKGROUND}

Pancreatic cancer (PC) is one of the most aggressive forms of cancer, and metastasis is the major cause of death (Garrido-Laguna and Hidalgo, 2015; Avula et al., 2020). To achieve a better treatment of $\mathrm{PC}$, it is urgent to understand the mechanisms that drive tumorigenesis, progression and metastasis.

Epithelial-mesenchymal transition (EMT) refers to a process by which epithelial cells lose cell-cell adhesion, and gain migratory and invasive properties (Dongre and Weinberg, 2019). EMT plays a key role during the progression of epithelial cancers to metastatic cancers 
(Dongre and Weinberg, 2019). SOX4 is a member of the SOX (SRY-related HMG-box) family of transcription factors and increased SOX4 expression contributes to cellular transformation and EMT in many cancer types ( $\mathrm{Li}$ et al., 2016; Liu et al., 2017; Zhang et al., 2020). SOX4 has a central role in promoting EMT, tumor growth and metastasis by directly regulating the expression of many genes involved in tumorigenesis, EMT, metastasis and cancer stemness (Hanieh et al., 2020). $\mathrm{EZH} 2$ is a catalytic subunit of the polycomb repressive complex 2, which catalyzes the trimethylation of histone3 lysine27 and mediates gene silencing (Chang and Hung, 2012). Inhibition of EZH2 attenuated EMT and metastasis in PC (Mody et al., 2016). It has been demonstrated that SOX4 functions directly as a transcriptional activator of the EZH2 promoter and induces the expression of EZH2 in cancer cells (Tiwari et al., 2013).

MicroRNAs (MiRNAs) are endogenously expressed small non-coding RNAs that regulate gene expression by binding to the $3^{\prime}$-untranslated regions (3'-UTRs) of specific target messenger RNAs, resulting in mRNA degradation or inhibition of translation (Xu et al., 2019). MiRNAs have also been proved to modulate the EMT process in cancer cells (Zhang and Ma, 2012)12]. On the other hand, circular RNAs (circRNAs) are highly stable forms of non-coding RNAs that, unlike linear RNAs, form a covalently closed continuous loop (Dragomir and Calin, 2018). Emerging studies have shown that circRNAs function as miRNA sponges to trap miRNAs in human tumor cells (Dong P. et al., 2020). It has been reported that the expression of circ_0001666 was elevated in PC tissues compared with normal tissues (Song et al., 2020). To date, however, the functions and underlying mechanisms of dysregulated circ_0001666 expression in the EMT process of human PC remain unknown.

In this study, we discovered that the expression of circ_0001666 was significantly upregulated in PC tissues and positively correlated with worse patient prognosis. We demonstrated that circ_0001666 works as a sponge for miR-1251 to promote EMT and invasion of PC cells, at least in part via upregulating the levels of SOX4, a target of miR-1251. Our findings indicated that the circ_0001666/miR-1251/SOX4 signaling pathway was highly associated with the aggressive phenotypes of PC cells, thus providing a potential therapeutic strategy for treating PC.

\section{MATERIALS AND METHODS}

\section{PC Patient Specimens}

A cohort of PC tissues and corresponding adjacent normal tissues were derived from 50 patients with PC who underwent surgery without preoperative chemotherapy or radiotherapy at Tongji University School of Medicine. All specimens were snap-frozen in liquid nitrogen upon collection. This study was approved by the Research Ethics Committee of Shanghai Pulmonary Hospital, Tongji University School of Medicine. Written informed consent was obtained from the patients before the study began.

\section{Cell Culture and Transient Transfection}

The human PC cell lines (AsPC-1, PANC-1, SW-1990 and PaCa-2) and the immortalized human pancreatic duct epithelial cell line HPDE6-C7 were obtained from the American Type Culture Collection (Manassas, VA, United States). All cell lines were maintained in RPMI1640 medium (Invitrogen, Carlsbad, CA, United States) supplemented with 10\% fetal bovine serum (FBS, Invitrogen). These cells were kept in a $37^{\circ} \mathrm{C}$ incubator with $5 \% \mathrm{CO} 2$.

Cells were transfected with Lipofectamine 3,000 reagent (Invitrogen). The vectors overexpressing circ_0001666 (or SOX4), and the control vector were purchased from Genepharma (Shanghai, China). SiRNAs against circ_0001666 (or SOX4), the miR-1251 mimic and miR-1251 inhibitor were purchased from Ribobio (Guangzhou, China).

\section{RNA Extraction and Real-Time PCR (qRT-PCR)}

Total RNA from cells and freshly frozen tissues was isolated using TRIzol reagent (Invitrogen). The total RNA was reverse transcribed using the PrimeScript RT Master Mix (Takara, Dalian, China). Total RNA was incubated at $37^{\circ} \mathrm{C}$ with $3 \mathrm{U} / \mu \mathrm{g}$ RNase R (Geneseed, Guangzhou, China) for $30 \mathrm{~min}$. Nuclear and cytoplasmic RNA fractionation was isolated using NE-PER Nuclear and Cytoplasmic Extraction Reagents (Thermo Fisher Scientific, CA. United States). The expression of circ_0001666 and SOX4 was quantified using SYBR Pre-mix Ex Taq quantitative PCR kit (Takara, Dalian, China). GAPDH was used as an internal control for circ_0001666 and SOX4. The expression of miR-1251 was determined using the mirVanaTM qRT-PCR microRNA Detection Kit (Ambion, Austin, TX, United States) and normalized to U6. The primer sequences were obtained from Ribobio (Guangzhou, China).

\section{Western Blotting Analysis}

PC cells were lysed with a RIPA buffer (Beyotime, Beijing, China). Equal amounts $(30 \mu \mathrm{g})$ of protein were separated on 15\% SDS-PAGE gels, and transferred onto PVDF membranes (Millipore, Bedford, MA, United States). The membranes were incubated with the primary antibodies, including anti-E-cadherin (Cell Signaling, MA, United States), anti-Vimentin (Cell Signaling), anti-EZH2 (Cell Signaling), antiSOX4 (Cell Signaling), and anti- $\beta$-actin (Cell Signaling) at $4^{\circ} \mathrm{C}$ overnight. Afterward, the secondary antibodies were added and blot signals were visualized with an ECL detection system (Amersham Biosciences, Buckinghamshire, United Kingdom).

\section{Cell Proliferation Assay}

Cell proliferation was detected by Cell Counting Kit-8 assay (CCK-8, Dojindo, Kumamoto, Japan). The cells were plated in 96-well plates. After 96-h incubation, $10 \mu \mathrm{l}$ CCK-8 solutions were added and the absorbance at $450 \mathrm{~nm}$ was determined using a microplate reader (Tecan Austria GmbH 5082, Grodig, Austria). 


\section{Invasion Assay}

For the transwell invasion assay, PC cells suspended in serumfree medium were seeded in the upper chambers $(8-\mu \mathrm{m}$ pore size, Corning Costar Co, CA, United States). $750 \mu \mathrm{l}$ medium containing $10 \%$ FBS was placed in the lower chamber, as previously reported (Dong et al., 2018). After incubation for $24 \mathrm{~h}$, the cells were fixed, stained and counted using an inverted microscope.

\section{Animal Experiment}

All animal experiments were approved by the Institutional Review Board of Shanghai Pulmonary Hospital, Tongji University School of Medicine. Four-week-old nude mice were purchased from Beijing HFK Bioscience (Beijing, China), and randomly divided into two groups $(n=5)$. PC cells transfected with circ_0001666 plasmid (or control vector), or with circ_0001666 siRNA (or control siRNA), were subcutaneously injected into the right flank of nude mice, respectively. The volume of the tumors was measured every week after implantation. The tumor volume was calculated using the following formula: tumor volume $\left[\mathrm{mm}^{3}\right]=$ (length $[\mathrm{mm}]) \times(\text { width }[\mathrm{mm}])^{2} \times 0.5$. The mice were sacrificed after 21 days. The tumors were removed, and the tumor weight was measured.

\section{Dual-Luciferase Reporter Assay}

The luciferase reporter plasmids containing wild-type (WT) circ_0001666 fragment, mutant (MUT) circ_0001666 fragment with a mutated miR-1251 binding site, WT SOX4 3'-untranslated region (3'-UTR) fragment, or MUT SOX4 3'-UTR fragment with a mutated miR-505 binding site, were purchased from Ribobio (Guangzhou, China). PC cells were co-transfected with the reporter plasmids with miR-1251 mimic, miR-1251 inhibitor or the respective negative controls, along with the Renilla luciferase plasmid pRL-CMV (Promega, WI, United States), using the Lipofectamine 3,000 (Invitrogen). The luciferase activities were measured using the Dual-Luciferase Reporter Assay System (Promega). Firefly luciferase activities were normalized to Renilla luciferase activities.

\section{RNA Immunoprecipitation Assay}

RNA Immunoprecipitation (RIP) assay was conducted with Magna RIP RNA-Binding Protein Immunoprecipitation Kit (Millipore, MA, United States). PC cells were lysed in a RIP-lysis buffer. Then, the magnetic beads conjugated with an anti-Ago2
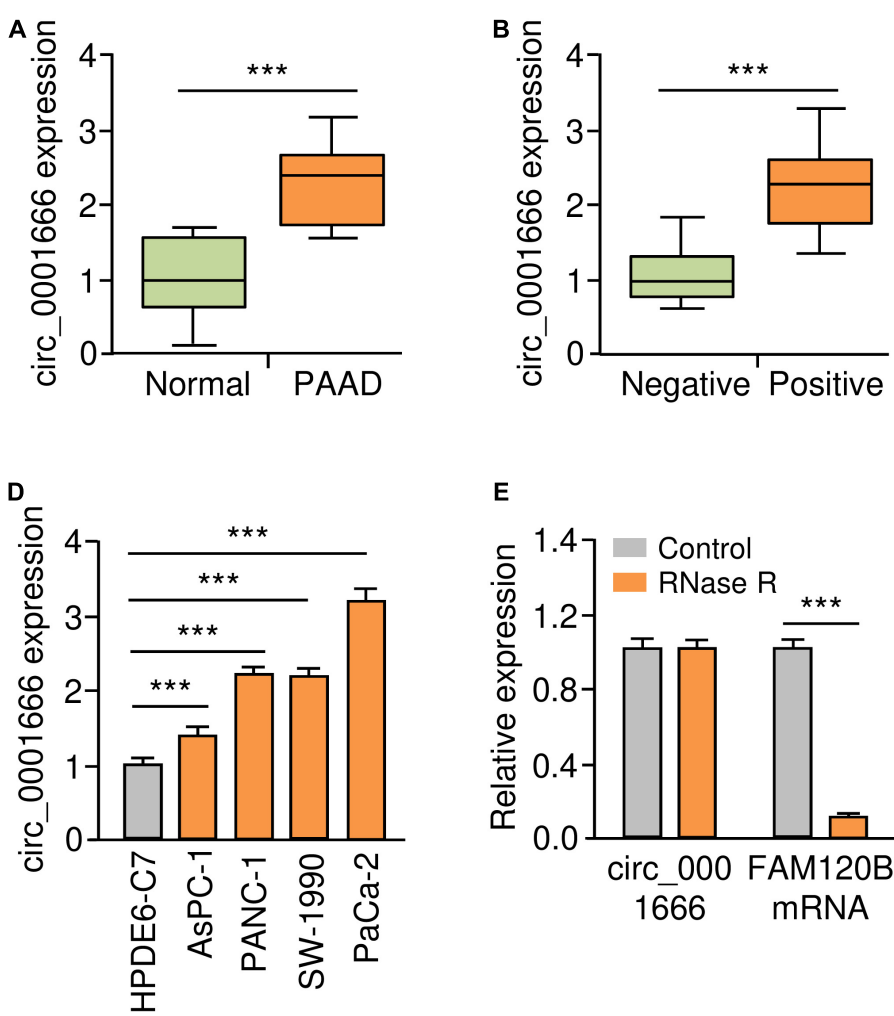

E
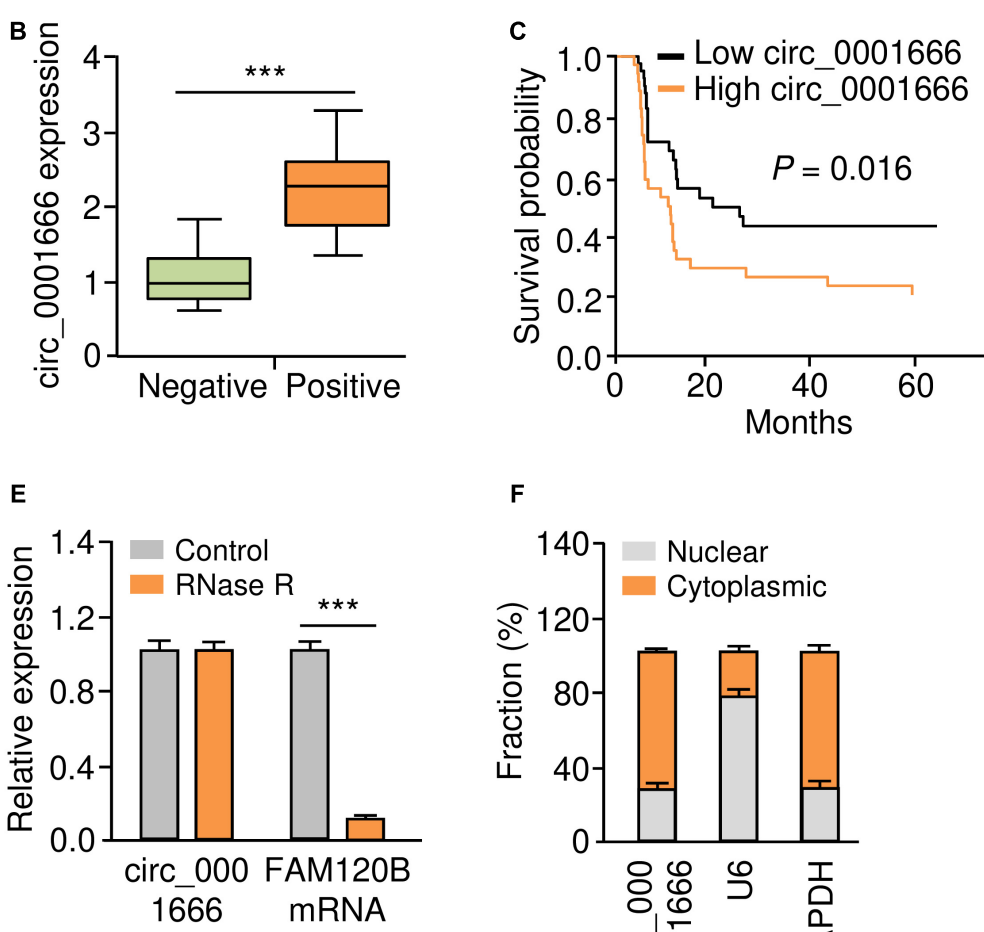

$\mathbf{F}$

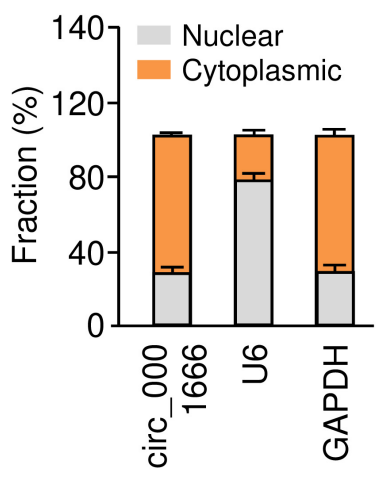

FIGURE 1 | Upregulation of circ_0001666 in PC tissues and PC cells. (A) The expression of circ_0001666 between PC tissues and adjacent normal tissues were assessed using qRT-PCR analysis. (B) qRT-PCR analysis of circ_0001666 expression in PC tissues from patients with or without lymph node metastasis. (C) Kaplan-Meier curves showed that higher expression of circ_0001666 was associated with poor overall survival in PC patients. (D) qRT-PCR assay of circ_0001666 expression in multiple PC cell lines and a normal pancreatic cell line HPDE6-C7. (E) qRT-PCR analysis of circ_0001666 and FAM120B expression in PaCa-2 cells in the presence or absence of RNase treatment. (F) Cytoplasmic and nuclear fractionation assay showed that circ_0001666 was mainly located in the cytoplasm. ${ }^{\star} P<0.05,{ }^{\star \star} P<0.01,{ }^{\star \star \star} P<0.001$. 
antibody (Millipore) or a control anti-IgG antibody (Millipore) were added to cell lysates, and the lysates were rotated overnight. After incubating with proteinase $\mathrm{K}$ for $30 \mathrm{~min}$, RNAs were purified and the levels of circ_0001666 and miR-1251 were quantified using qRT-PCR analysis.

\section{Statistical Analysis}

All results were representative results from at least three independent experiments. Data are expressed as the mean \pm standard deviation. Significant differences for the mean values between groups were determined using Student's $t$-tests and one-way ANOVA. Data analysis was performed by using SPSS 25.0 software. $P<0.05$ was considered statistically significant.

\section{RESULTS}

\section{Upregulation of circ_0001666 in PC Tissues and PC Cells}

According to the annotation of circBase database ${ }^{1}$, we found that FAM120B is the host gene of circ_0001666. Circ_0001666 is located at chr6:170626457-170639638, and the length of

${ }^{1}$ http://www.circbase.org/ its mature transcript is $2038 \mathrm{nt}$. In order to understand the role of circ_0001666 in PC, we used qRT-PCR assay to examine the expression of circ_0001666 in 50 pairs of primary PC tissues and adjacent non-cancerous tissues derived from PC patients. We found that circ_0001666 was significantly overexpressed in PC samples compared with adjacent normal tissues (Figure 1A). We next evaluated the association between circ_0001666 expression and clinicalpathological parameters, and found that higher expression of circ_0001666 was positively correlated with the presence of lymph node metastasis (Figure 1B). Survival analysis revealed that those patients with high (above the median) circ_0001666 levels displayed lower overall survival rates (Figure 1C).

The level of circ_0001666 in four PC cell lines and one normal pancreatic duct epithelial cell line HPDE6C7 was further confirmed by qRT-PCR assay. As expected, circ_0001666 were consistently upregulated in PC cell lines compared with HPDE6-C7 cells (Figure 1D). As shown in Figure 1E, circ_0001666 resisted the digestion of RNase R, while the linear form of FAM $120 B$ mRNA was digested by RNase sharply. We detected the location of circ_0001666 in PC cells, and found that this circRNA was mainly localized in the cytoplasm (Figure $\mathbf{1 F}$ ). These results show that circ_0001666 is overexpressed in PC tissues and cell lines,

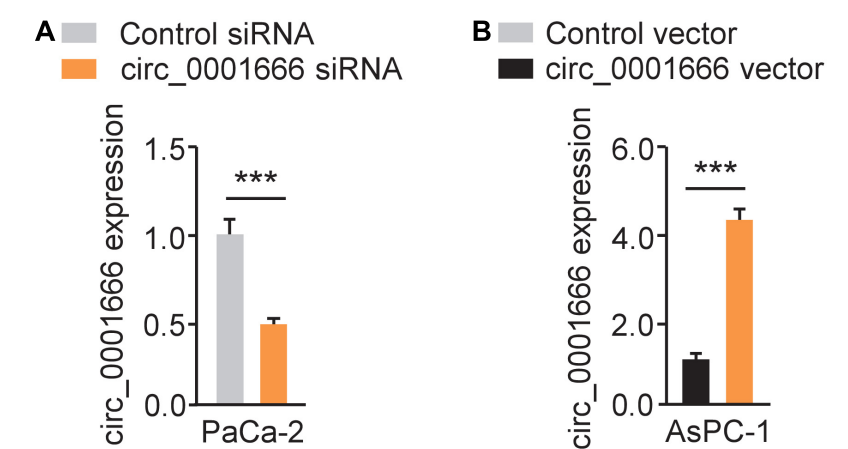

C Control siRNA
circ_0001666 siRNA

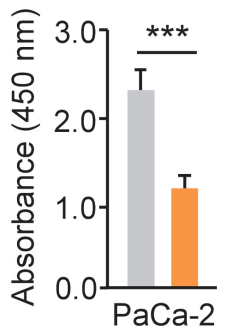

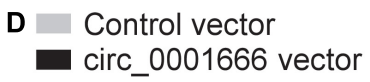

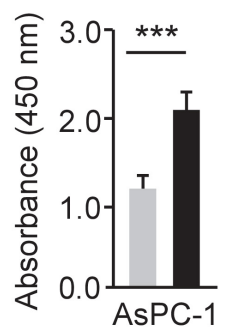

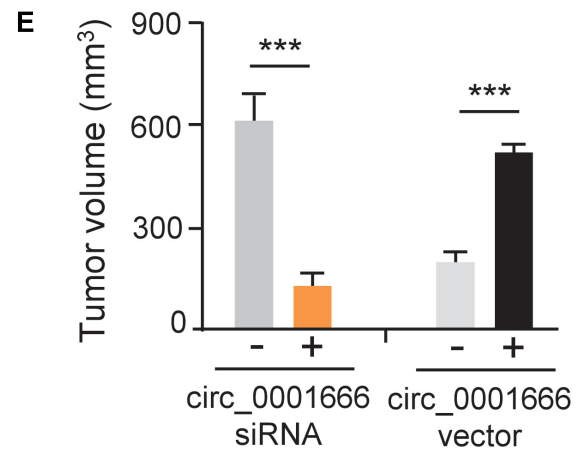

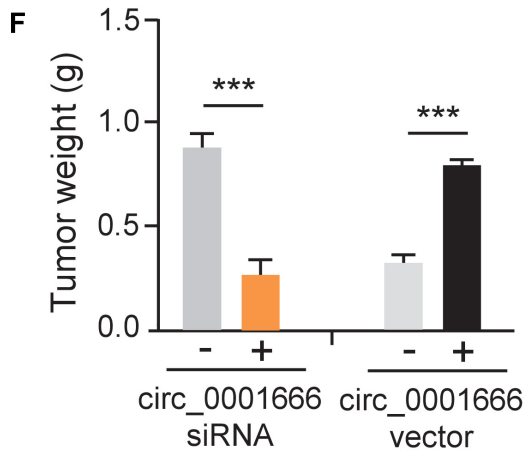

FIGURE 2 | Knockdown of circ_0001666 inhibits the proliferation of PC cells. (A) qRT-PCR assay of circ_0001666 expression in PaCa-2 cells transfected with circ_0001666 siRNA or control siRNA. (B) qRT-PCR assay of circ_0001666 expression in AsPC-1 cells transfected with circ_0001666 vector or control vector. (C,D) CCK-8 assay in PaCa-2 (C) or AsPC-1 (D) cells transfected as indicated. (E,F) The tumor volume (E) and tumor weight (F) of the tumors derived from PaCa-2 or AsPC-1 cells. ${ }^{\star \star \star} P<0.001$. 

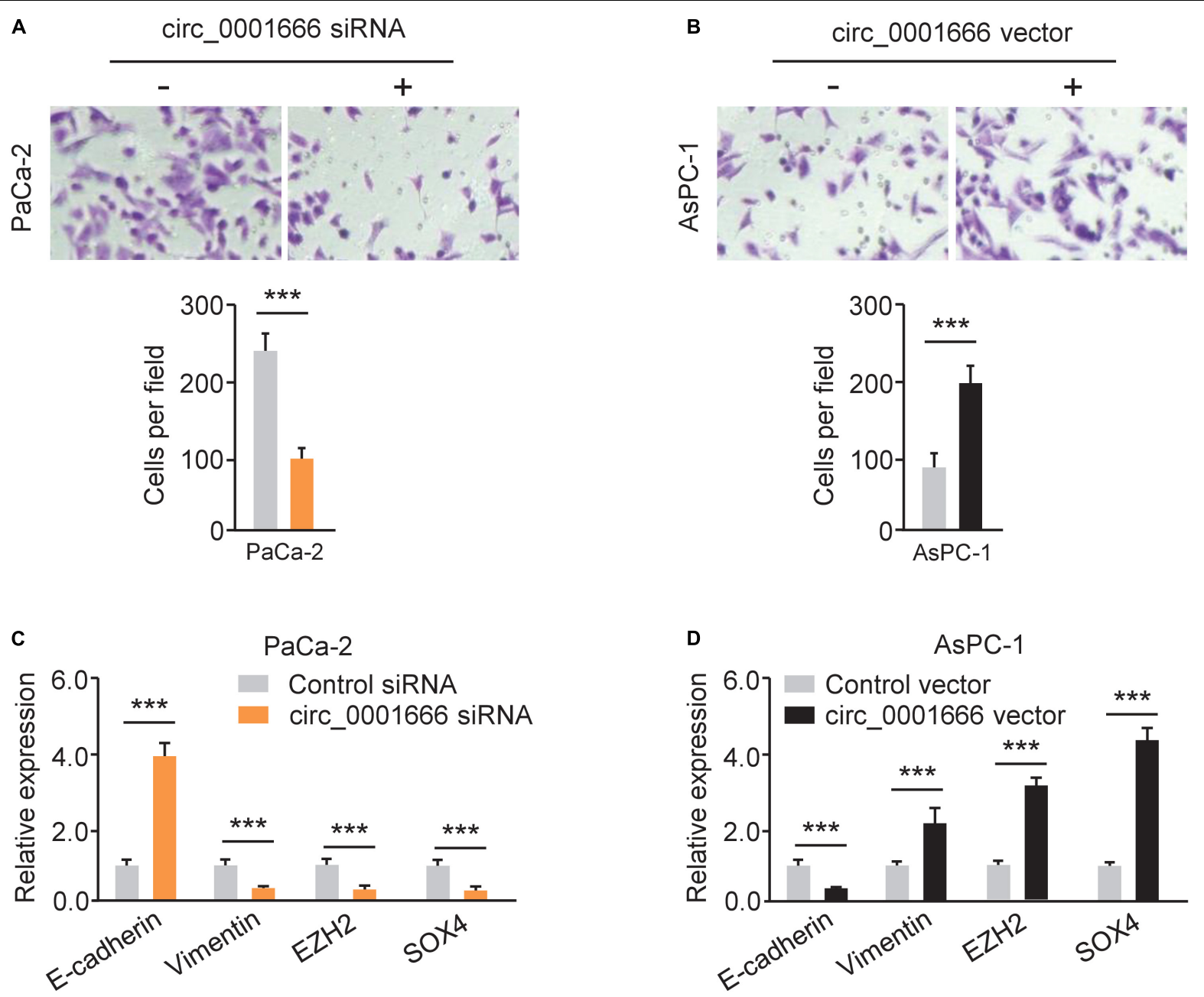

D AsPC-1

$\mathbf{E}$

PaCa-2

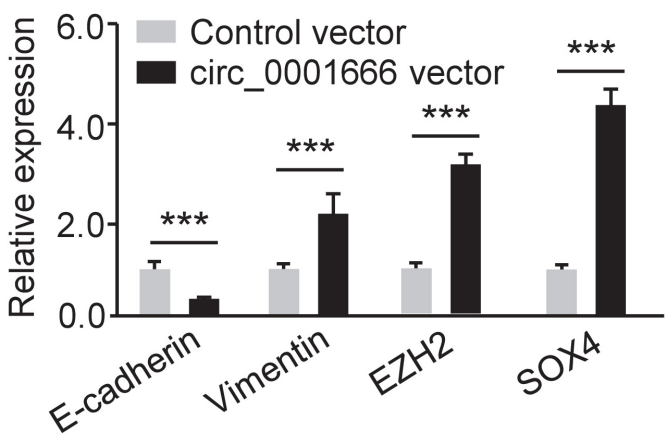

$\mathbf{F}$

\section{AsPC-1}
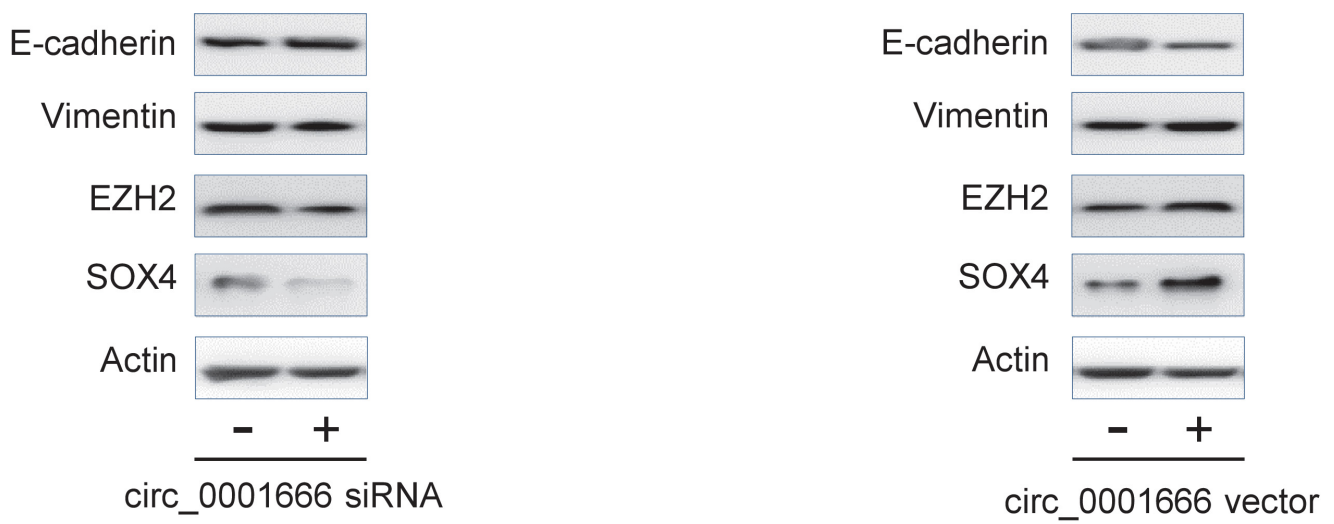

FIGURE 3 | Knockdown of circ_0001666 represses EMT in PC cells. (A) The invasive abilities of PaCa-2 cells following knockdown of circ_0001666 were evaluated using cell invasion assay. (B) The invasive abilities of AsPC-1 cells following overexpression of circ_0001666 were evaluated using cell invasion assay. (C,D) qRT-PCR analysis of EMT-related genes in PaCa-2 cells following knockdown of circ_0001666 (C), and in AsPC-1cells following overexpression of circ_0001666 (D). (E,F) Western blotting analysis of EMT-related genes in PaCa-2 cells following knockdown of circ_0001666 (E), and in AsPC-1cells following overexpression of circ_0001666 (F). ${ }^{\star \star \star} P<0.001$. 
and high circ_0001666 expression is correlated with poor prognosis in PC patients.

\section{Knockdown of circ_0001666 Inhibits the Proliferation of PC Cells}

We characterized the role of circ_0001666 in PC cells. Circ_0001666 knockdown and overexpression models were constructed in PaCa-2 (Figure 2A) cells and AsPC-1 cells (Figure 2B), respectively. The knockdown or overexpression efficiency was validated by qRT-PCR assay (Figure 2B). Then, the cell proliferation rate in these cells was tested using CCK8 assay. Knockdown of circ_0001666 significantly reduced the proliferation of PaCa-2 cells (Figure 2C), and overexpression of circ_0001666 significantly improved the proliferation of AsPC-1 cells (Figure 2D). In addition, to evaluate the role of circ_0001666 in tumor growth in vivo, nude mice were injected with PaCa-2 cells that were transfected with circ_0001666 siRNA or control siRNA, and with AsPC-1 cells that were transfected with circ_0001666 overexpression vector or the control vector. The volume (Figure 2E) and weight (Figure 2F) of tumors were markedly lower in circ_0001666 knockdown group than the control group, while the volume (Figure 2E) and weight (Figure 2F) of tumors were significantly higher in circ_0001666 overexpression group than the control group. Our results suggest that knockdown of circ_0001666 inhibits PC growth both in vitro and in vivo.

\section{Knockdown of circ_0001666 Represses EMT in PC}

EMT is one of the major mechanisms for the enhanced invasion ability of cancer cells (Dongre and Weinberg, 2019). To evaluate the potential effects of circ_0001666 on EMT, we performed a cell invasion assay and demonstrated that knockdown of circ_0001666 significantly inhibited cell invasion in PaCa-2 cells (Figure 3A). However, the invasive abilities of AsPC-1 cells were significantly enhanced following overexpression of circ_0001666 (Figure 3B). Furthermore, the mRNA expression of EMT markers in circ_0001666-silenced PaCa-2 cells or circ_0001666-overexpressing AsPC-1 cells was investigated using qRT-PCR assay. The expression of E-cadherin was increased, while the expression of Vimentin was decreased in circ_0001666silenced PaCa-2 cells (Figure 3C). Consistently, overexpression of circ_0001666 led to the downregulation of E-cadherin and upregulation of Vimentin in AsPC-1 cells (Figure 3D). These results were verified using western blotting analysis (Figures 3E,F). Our data suggest the role of circ_0001666 in promoting EMT in PC cells.

\section{Circ_0001666 Acts as a miRNA Sponge of miR-1251 to Promote PC Cell Invasion}

Here, we explored whether circ_0001666 promotes PC progression by sponging miRNAs. First, we predicted the potential miRNAs that might bind to circ_0001666 using the CircInteractome database. Circ_0001666 had a complementary binding sequence to miR-1251 (Figure 4A). The expression of miR-1251 in PC tissues was lower than that in the adjacent normal tissues (Figure 4B). Meanwhile, reduced expression of miR-1251 was observed in multiple PC cell lines compared with HPDE6-C7 cells (Figure 4C). The survival curves from KaplanMeier Plotter database showed that lower levels of miR-1251 predicted a poorer outcome in PC patients (Figure 4D).

The luciferase reporter assays illustrated that overexpression of miR-1251 suppressed the luciferase activity of WT circ_0001666 sequence, and knockdown of miR-1251 increased the luciferase activity of WT circ_0001666 sequence (Figures 5A,B). However, modulation of miR1251 expression did not change the luciferase activity of mutated circ_0001666 sequence (Figures 5A,B), suggesting
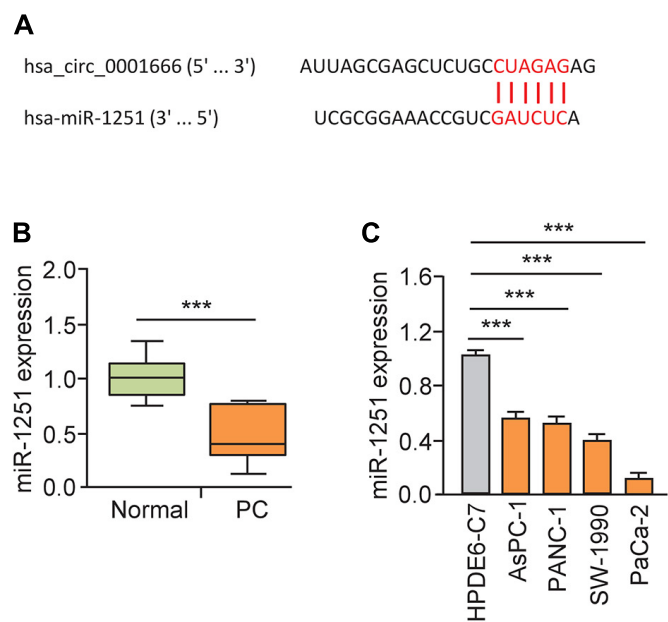

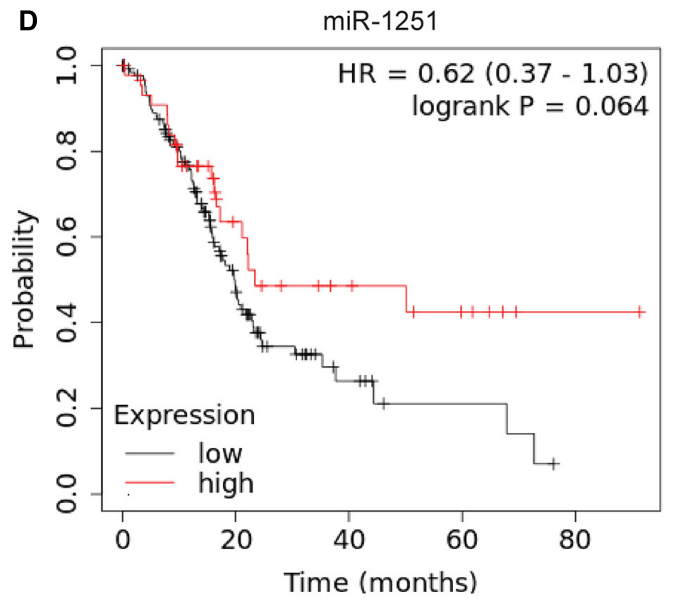

FIGURE 4 | MiR-1251 is downregulated in PC tissues and associated with a better prognosis. (A) The predicted miR-1251 binding site in the circ_0001666 sequence. (B) The expression of miR-1251 in PC tissues and adjacent normal tissues, as measured by qRT-PCR assay. (C) qRT-PCR analysis of miR-1251 expression in PC cell lines and normal pancreatic cells. (D) The prognostic role of miR-1251 expression in PC was explored using the KM plotter database. ${ }^{\star \star \star} P<0.001$. 
a direct interaction between circ_0001666 and miR-1251 in PC cells. Subsequent RIP assay was used to pull down circ_0001666 and miR-1251 with an anti-AGO2 antibody (IgG as a negative control) in PaCa-2 and AsPC-1 cells. We found that both circ_0001666 and miR-1251 were efficiently pulled down by anti-AGO2 antibody compared with anti-IgG (Figure 5C). These results demonstrated that circ_0001666 binds to miR-1251 in PC cells.

Furthermore, knockdown of circ_0001666 upregulated the expression of miR-1251, while overexpression of circ_0001666

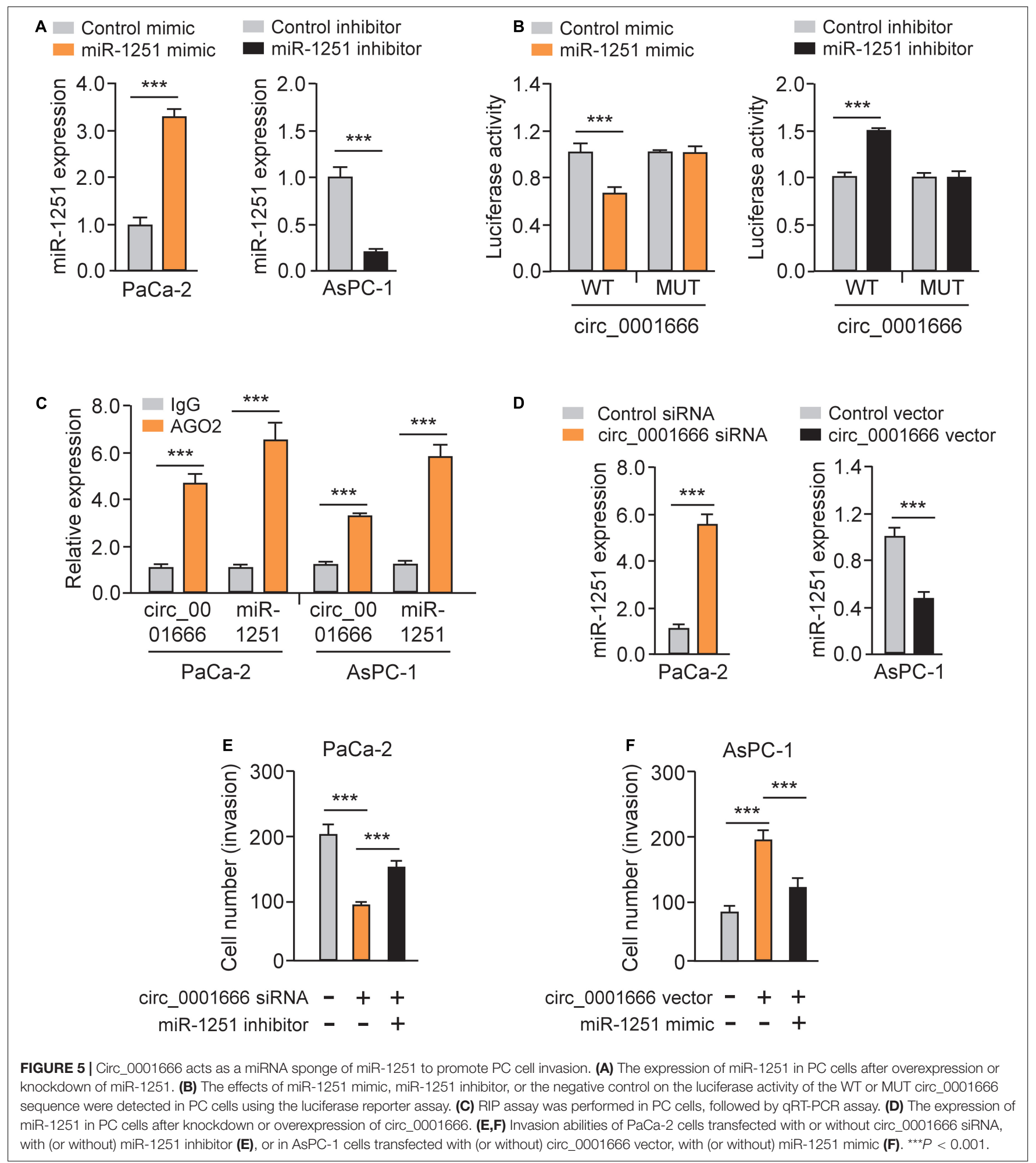


displayed an opposite effect (Figure 5D). Importantly, cotransfection of miR-1251 inhibitor could rescue cell invasion that was suppressed by circ_0001666 knockdown (Figure 5E).
Additionally, overexpression of circ_0001666 led to increased cell invasion, but this change was reversed by ectopic expression of miR-1251 (Figure 5F). Taken together, our results suggest

A

Position 1629-1635 of SOX4 3'-UTR

hsa-miR-1251-5p
5' ...CUCCUUCACUGAAGgGCUAGAGU...

| | | | | | |

3' UCGCGGAAACCGUCGAUCUCA
B
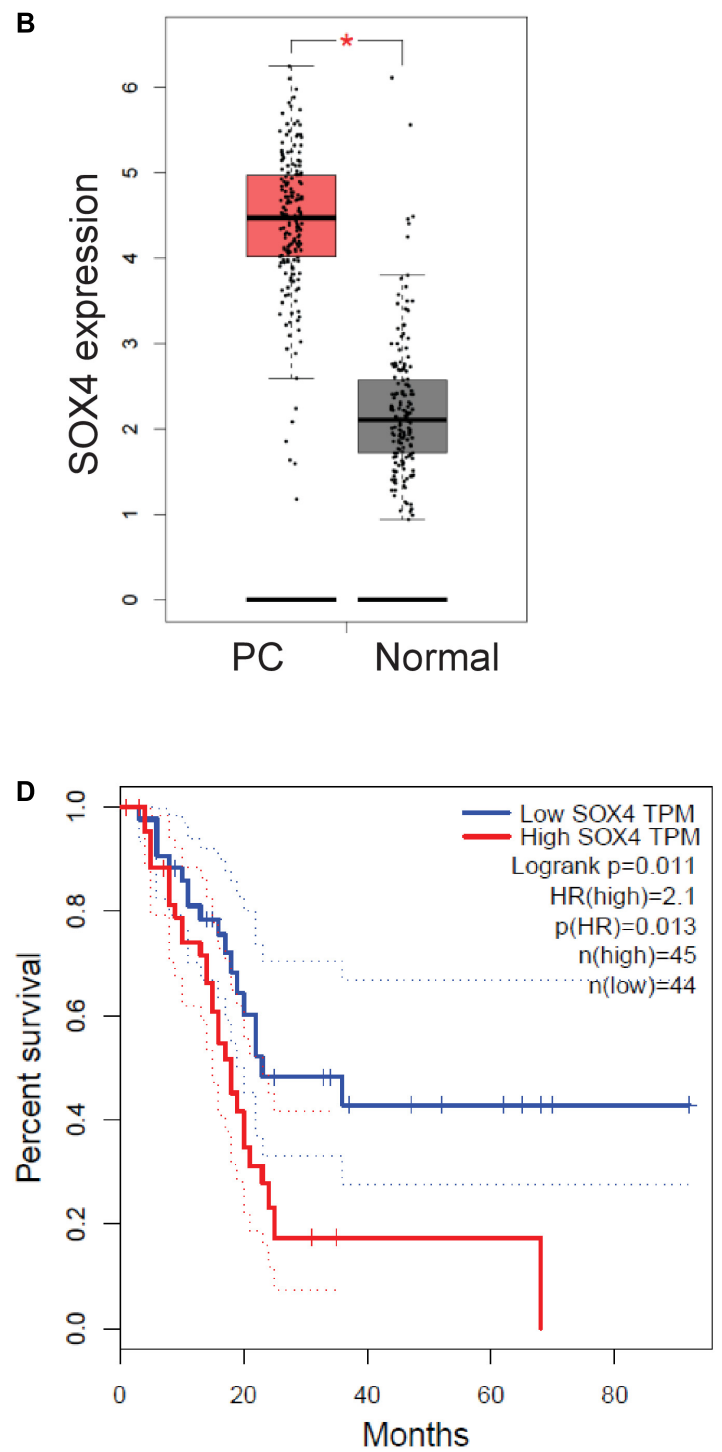

C
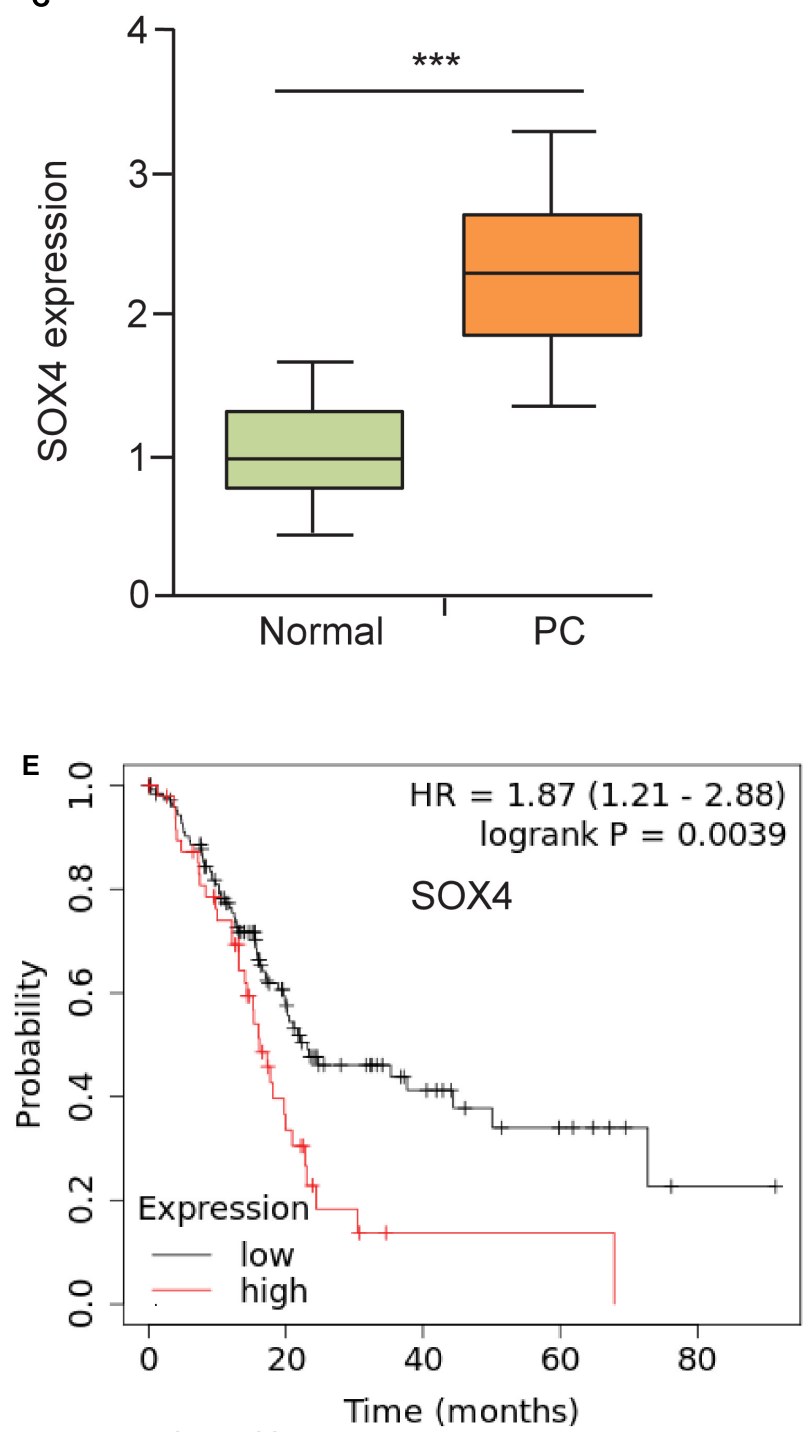

FIGURE 6 | Upregulation of SOX4 is correlated with worse patient survival. (A) Predicted binding site between miR-1251 and SOX4 (TargetScan database). (B) The expression of SOX4 expression in PC tissues and adjacent normal tissues (GEPIA database). (C) SOX4 mRNA expression in PC tissues and adjacent normal tissues was tested using qRT-PCR assay. (D,E) Kaplan-Meier curves showed the overall survival of PC patients expressing higher or lower SOX4 expression (D: GEPIA database; E: KM Plotter database). ${ }^{* \star \star} P<0.001$. 
that circ_0001666 acts as a sponge of miR-1251 to promote PC cell invasion.

\section{MiR-1251 Regulates EMT and PC Cell Invasion by Targeting SOX4}

To explore the functional downstream target of miR-1251, we predicted the possible binding sites using the TargetScan database. We found that SOX4 might be a target of miR1251 (Figure 6A). Using the GEPIA database, we explored SOX4 mRNA expression between PC and normal tissues. The results demonstrated that the expression of SOX4 was significantly higher in PC tissues (Figure 6B). We confirmed the overexpression of S100A in PC tissues using qRT-PCR assay (Figure 6C). We next used GEPIA and Kaplan-Meier plotter databases to analyze the prognostic values of the mRNA expression of SOX4 in PC patients. Higher mRNA expression of SOX4 was significantly associated with shorter overall survival of PC patients (Figures 6D,E).

As expected, we observed a significantly high expression of SOX4 in PC cell lines compared to normal cells (Figure 7A). Since SOX4 regulates EMT of cancer cells by controlling EZH2 expression (Hanieh et al., 2020), we investigated whether
miR-1251 could modulate the protein expression of EMT-related markers, SOX4 and EZH2 in PC cells. Our western blotting assay showed that overexpression of miR-1251 significantly enhanced the levels of E-cadherin, but downregulated the expression of Vimentin, SOX4 and EZH2 (Figure 7B). Knockdown of miR-1251 had opposite effects (Figure 7B). Moreover, our rescue experiments demonstrated that the ability of miR-1251 in regulating the expression of these proteins was largely abrogated by overexpression of SOX4, and the impact of miR1215 inhibition on gene expression was reversed by transfection with SOX4 siRNA (Figure 7B).

Luciferase reporter assays were performed and the results showed that transfection with miR-1251 mimic significantly decreased the luciferase activity of the WT SOX $43^{\prime}$-UTR, while the introduction of miR-1251 inhibitor could remarkably increase it, but not that of the MUT SOX4 $3^{\prime}$-UTR in PC cells (Figure 7C). These data clearly suggested that miR-1251 suppresses SOX4 expression in PC cells by directly binding to 3'-UTR of SOX4 mRNA. Subsequent rescued experiments have shown that overexpression of SOX4 significantly reversed the ability of miR-1251 to suppress the invasion of $\mathrm{PaCa}-2$ cells (Figure 7D). In line with the role of circ_0001666 as an upstream regulator of miR-1251, our western blotting experiments revealed
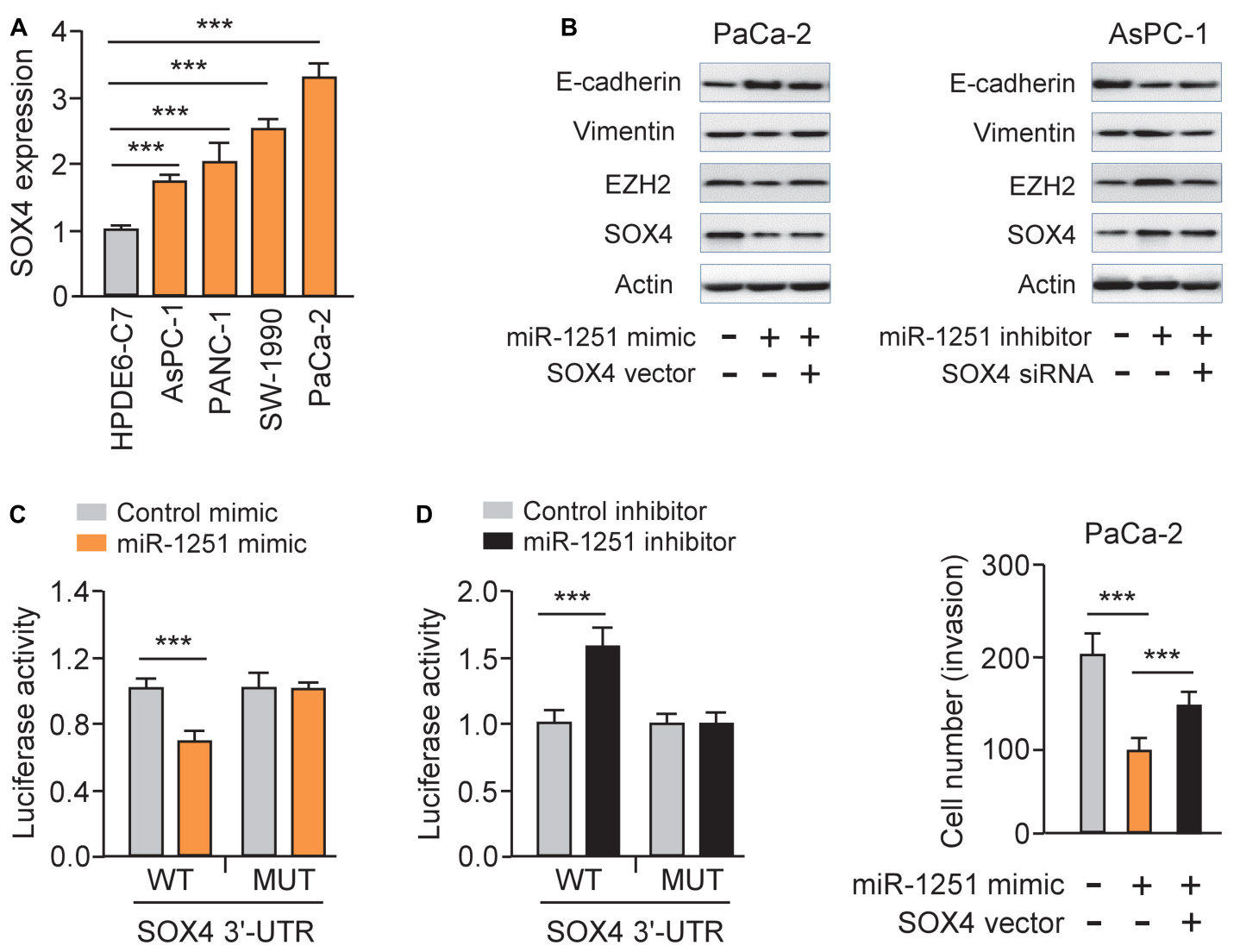

FIGURE 7 | MiR-1251 regulates EMT and PC cell invasion by targeting SOX4. (A) qRT-PCR assay of SOX4 expression in PC cell lines and a normal pancreatic cell line HPDE6-C7. (B) Western blotting analysis of E-cadherin, Vimentin, EZH2, SOX4, and $\beta$-actin expression in PC cells transfected as indicated. (C) Luciferase reporter assay for luciferase activities of the WT or MUT SOX4 $3^{\prime}-U T R$ reporter in PC cells transfected as indicated. (D) Cell invasion assay in PC cells transfected as indicated. ${ }^{\star \star \star} P<0.001$. 

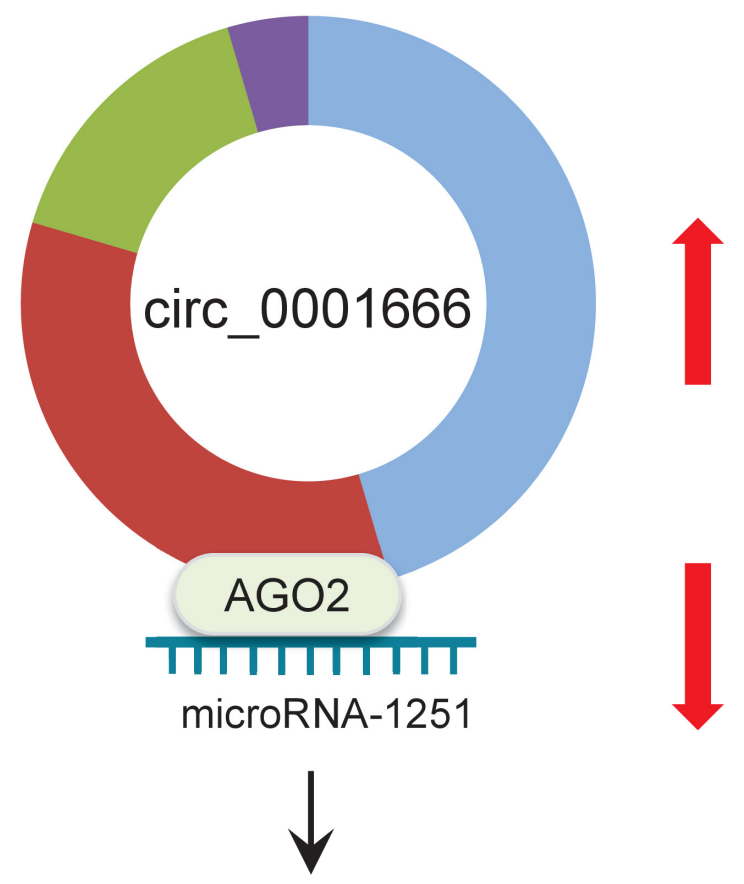

SOX4 mRNA

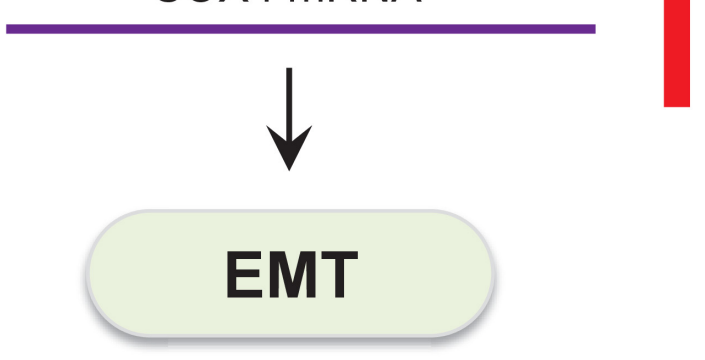

FIGURE 8 | Schematic representation of the proposed mechanism by which circ_0001666 mediates EMT in PC cells. Circ_0001666 acts as a miR-1251 sponge to upregulate the expression of SOX4, thereby promoting EMT and $\mathrm{PC}$ cell invasion.

that overexpression of circ_0001666 resulted in the upregulation of SOX4 and EZH2 in AsPC-1 cells (Figure 3E). However, silencing of circ_0001666 downregulated the protein expression of SOX4 and EZH2 (Figure 3E). These results support the notion that circ_0001666 increased the expression of SOX4 and EZH2 by downregulating miR-1251 expression.

In summary, all of these results indicated that circ_0001666 promotes EMT and invasive properties of PC cells through sponging miR-1251 and enhancing SOX4 expression (Figure 8).

\section{DISCUSSION}

Recently, larger numbers of circRNAs have been discovered by RNA sequencing, and many of the differentially expressed circRNAs were shown to have oncogenic or tumor suppressor functions in vitro and in vivo (Dong P. et al., 2020).
Altered expression of circRNA is closely related to oncogenesis, progression and metastasis in PC (Rong et al., 2021). Although the upregulation of circ_0001666 has already been recognized in PC tissues (Song et al., 2020), its functional relevance and molecular mechanisms remain undetermined. In this study, we verified the overexpression of circ_0001666 in PC samples and found that high circ_0001666 expression was associated with poor prognosis in PC patients. The small sample size was a limitation of our study. Our future research based on large sample sizes should be performed to evaluate the clinical significance of circ_0001666 in PC patients. Furthermore, we showed that circ_0001666 works as a sponge for miR-1251 to induce EMT and invasion of PC cells, at least in part via upregulating the levels of SOX4, a target of miR-1251. Our results suggest that circ_0001666 is acting as an oncogenic circRNA through upregulation of SOX4 by inhibiting miR1251 expression.

Most studies have validated that circRNAs are functional transcripts that could regulate gene expression through modulating gene transcription and splicing, titrating miRNAs, interacting with proteins, and acting as templates for the synthesis of polypeptides (Chen, 2020). Many circRNAs accumulate in the cytoplasm and function as competing endogenous RNAs, which can bind and sponge target miRNAs to prevent these miRNAs from binding and suppressing their target mRNAs (Chen, 2020). For example, elevated expression of circ_0066147 enhanced the proliferation, invasion, migration, and EMT process of PC cells in vitro by sponging miR-330-5p (Xu et al., 2020). Circ_0006948 is a sponge of miR-490 in esophageal squamous cell carcinoma cells, and it upregulates the expression of oncogene HMGA2 to induce EMT by sequestering miR-490 (Pan et al., 2019). These findings are in accordance with our observations that, in PC cells, circ_0001666 functions as a competing endogenous RNA to decrease miR-1251 expression, thus promoting SOX4-dependent EMT. On the other hand, the function of circRNA as protein scaffold or sequester protein has been exemplified by numerous examples (Chen, 2020). CircPABPN1 is reported to sequester HuR, thereby serving as a decoy for HuR and impairing PABPN1 translation in cervical cancer cells (Abdelmohsen et al., 2017). HuR recognizes and binds to the AU-rich elements in its target mRNAs, and enhances mRNA stability and translation (Mukherjee et al., 2011). HuR binds to the $3^{\prime}$-UTR of the mRNA of the EMT inducer Snail, resulting in stabilization of Snail mRNA and enhanced Snail protein expression and thus promoting EMT, metastasis, and formation of stem-like PC cells (Dong R. et al., 2020). Using the CircInteractome database, we have found that mature circ_0001666 hosts multiple binding sites for several RNAbinding proteins like HuR (data not shown). The association between circ_0001666 and HuR (or other proteins) in PC cells represents an interesting topic for future research.

The published studies showed that miR-1251 significantly affected the prognosis of PC patients and high expression of miR1251 is associated with favorable survival (Jia et al., 2020). In line with this data, we determined that miR-1251 impairs the EMT features of PC cells. Of note, miR-1251 was also reported to promote tumor growth and metastasis of hepatocellular 
carcinoma (Han et al., 2020), and facilitate carcinogenesis and autophagy in ovarian cancer (Shao et al., 2019). These data imply that that miR-1251 has complex roles in regulating tumor initiation and progression depending on cell type, and further characterization of its target genes would provide insights in miR-1251-reduced EMT in PC cells.

The SOX4 gene is frequently amplified and overexpressed in more than 20 types of malignancies, where SOX4 acts as an oncogene (Moreno, 2020). SOX4 interacts with multiple other transcription factors, rendering its impacts on gene expression and leading to the promotion of cell survival, cancer stemness, EMT and metastasis (Moreno, 2020). Importantly, knockdown of SOX4 with siRNA in PC cells resulted in restricted tumor growth both in vitro and in mice (Huang et al., 2012). In addition, miR-129 and miR-335 target SOX4 mRNA for degradation in PC cells (Huang et al., 2012). Our study added new evidence to support the oncogenic role of SOX4 in PC cells, and its overexpression is partially due to the repression of miR-1251. Thus, SOX4 might globally regulate the expression of many genes (such as EZH2) (Hanieh et al., 2020; Moreno, 2020), thereby contributing to EMT and PC metastasis.

In conclusion, we described a novel circ_0001666/miR$1251 /$ SOX 4 regulatory pathway in PC cells. Circ_0001666 represses miR-1251 expression to increase SOX4 expression, subsequently inducing EMT and enhancing PC cell invasion (Figure 8). Overall, the components of this pathway could be promising targets for developing new PC therapies.

\section{REFERENCES}

Abdelmohsen, K., Panda, A. C., Munk, R., Grammatikakis, I., Dudekula, D. B., De, S., et al. (2017). Identification of HuR target circular RNAs uncovers suppression of PABPN1 translation by CircPABPN1. RNA Biol. 14, 361-369. doi: 10.1080/15476286.2017.1279788

Avula, L. R., Hagerty, B., and Alewine, C. (2020). Molecular mediators of peritoneal metastasis in pancreatic cancer. Cancer Metastasis Rev. 39, 1223-1243. doi: 10.1007/s10555-020-09924-4

Chang, C. J., and Hung, M. C. (2012). The role of EZH2 in tumour progression. $\mathrm{Br}$ J Cancer. 106, 243-247. doi: 10.1038/bjc.2011.551

Chen, L. L. (2020). The expanding regulatory mechanisms and cellular functions of circular RNAs. Nat Rev Mol Cell Biol. 21, 475-490. doi: 10.1038/s41580-0200243-y

Dong, P., Xiong, Y., Yue, J., Hanley, S. J. B., and Watari, H. (2018). miR-34a, miR424 and miR-513 inhibit MMSET expression to repress endometrial cancer cell invasion and sphere formation. Oncotarget 9, 23253-23263. doi: 10.18632/ oncotarget. 25298

Dong, P., Xu, D., Xiong, Y., Yue, J., Ihira, K., Konno, Y., et al. (2020). The expression, functions and mechanisms of circular RNAs in gynecological cancers. Cancers (Basel) 12:1472. doi: 10.3390/cancers12061472

Dong, R., Chen, P., Polireddy, K., Wu, X., Wang, T., Ramesh, R., et al. (2020). An RNA-binding protein, hu-antigen $\mathrm{R}$, in pancreatic cancer epithelial to mesenchymal transition, metastasis, and cancer stem cells. Mol. Cancer Ther. doi: 10.1158/1535-7163.MCT-19-0822 [Epub ahead of print].

Dongre, A., and Weinberg, R. A. (2019). New insights into the mechanisms of epithelial-mesenchymal transition and implications for cancer. Nat. Rev. Mol. Cell Biol. 20, 69-84. doi: 10.1038/s41580-018-0080-4

Dragomir, M., and Calin, G. A. (2018). Circular RNAs in cancer - lessons learned from microRNAs. Front. Oncol. 8:179. doi: 10.3389/fonc.2018.00179

\section{DATA AVAILABILITY STATEMENT}

The original contributions presented in the study are included in the article/supplementary material, further inquiries can be directed to the corresponding author/s.

\section{ETHICS STATEMENT}

The studies involving human participants were reviewed and approved by the Research Ethics Committee of Tongji University School of Medicine. The patients/participants provided their written informed consent to participate in this study. The animal study was reviewed and approved by the Institutional Review Board of Tongji University School of Medicine.

\section{AUTHOR CONTRIBUTIONS}

KA designed the experiments. RZ performed the experiments. WZ and CM analyzed the data. All authors read and approved the final manuscript.

\section{FUNDING}

This study was supported by grants from the National Natural Science Foundation of China (No. 82072742).

Garrido-Laguna, I., and Hidalgo, M. (2015). Pancreatic cancer: from state-of-theart treatments to promising novel therapies. Nat. Rev. Clin. Oncol. 12, 319-334. doi: 10.1038/nrclinonc.2015.53

Han, S., Wang, L., Sun, L., Wang, Y., Yao, B., Chen, T., et al. (2020). MicroRNA1251-5p promotes tumor growth and metastasis of hepatocellular carcinoma by targeting AKAP12. Biomed. Pharmacother. 122:109754. doi: 10.1016/j.biopha. 2019.109754

Hanieh, H., Ahmed, E. A., Vishnubalaji, R., and Alajez, N. M. (2020). SOX4: epigenetic regulation and role in tumorigenesis. Semin. Cancer Biol. 67(Pt 1), 91-104. doi: 10.1016/j.semcancer.2019.06.022

Huang, H. Y., Cheng, Y. Y., Liao, W. C., Tien, Y. W., Yang, C. H., Hsu, S. M., et al. (2012). SOX4 transcriptionally regulates multiple SEMA3/plexin family members and promotes tumor growth in pancreatic cancer. PLoS One 7:e48637. doi: 10.1371/journal.pone.0048637

Jia, Y., Shen, M., Zhou, Y., and Liu, H. (2020). Development of a 12-biomarkersbased prognostic model for pancreatic cancer using multi-omics integrated analysis. Acta Biochim. Pol. 67, 501-508. doi: 10.18388/abp.2020_5225

Li, Y., Chen, P., Zu, L., Liu, B., Wang, M., and Zhou, Q. (2016). MicroRNA-338-3p suppresses metastasis of lung cancer cells by targeting the EMT regulator Sox4. Am. J. Cancer Res. 6, 127-140.

Liu, Y., Zeng, S., Jiang, X., Lai, D., and Su, Z. (2017). SOX4 induces tumor invasion by targeting EMT-related pathway in prostate cancer. Tumour. Biol. 39:1010428317694539. doi: 10.1177/1010428317694539

Mody, H. R., Hung, S. W., AlSaggar, M., Griffin, J., and Govindarajan, R. (2016). Inhibition of S-adenosylmethionine-dependent methyltransferase attenuates TGF $\beta 1$-induced EMT and metastasis in pancreatic cancer: putative roles of miR-663a and miR-4787-5p. Mol. Cancer Res. 14, 1124-1135. doi: 10.1158/ 1541-7786.MCR-16-0083

Moreno, C. S. (2020). SOX4: the unappreciated oncogene. Semin. Cancer Biol. 67(Pt 1), 57-64. doi: 10.1016/j.semcancer.2019.08.027 
Mukherjee, N., Corcoran, D. L., Nusbaum, J. D., Reid, D. W., Georgiev, S., Hafner, M., et al. (2011). Integrative regulatory mapping indicates that the RNA-binding protein HuR couples pre-mRNA processing and mRNA stability. Mol. Cell. 43, 327-339. doi: 10.1016/j.molcel.2011.06.007

Pan, Z., Lin, J., Wu, D., He, X., Wang, W., Hu, X., et al. (2019). Hsa_circ_0006948 enhances cancer progression and epithelial-mesenchymal transition through the miR-490-3p/HMGA2 axis in esophageal squamous cell carcinoma. Aging (Albany N. Y.) 11, 11937-11954. doi: 10.18632/aging.102519

Rong, Z., Xu, J., Shi, S., Tan, Z., Meng, Q., Hua, J., et al. (2021). Circular RNA in pancreatic cancer: a novel avenue for the roles of diagnosis and treatment. Theranostics 11, 2755-2769. doi: 10.7150/thno.56174

Shao, Y., Liu, X., Meng, J., Zhang, X., Ma, Z., and Yang, G. (2019). MicroRNA-1251$5 p$ promotes carcinogenesis and autophagy via targeting the tumor suppressor TBCC in ovarian cancer cells. Mol. Ther. 27, 1653-1664. doi: 10.1016/j.ymthe. 2019.06.005

Song, W., Wang, W. J., Fu, T., Chen, L., and Miao, D. L. (2020). Integrated analysis of circular RNA-associated ceRNA network in pancreatic ductal adenocarcinoma. Oncol. Lett. 19, 2175-2184. doi: 10.3892/ol.2020.11306

Tiwari, N., Tiwari, V. K., Waldmeier, L., Balwierz, P. J., Arnold, P., Pachkov, M., et al. (2013). Sox4 is a master regulator of epithelial-mesenchymal transition by controlling Ezh2 expression and epigenetic reprogramming. Cancer Cell 23, 768-783. doi: 10.1016/j.ccr.2013.04.020

Xu, D., Dong, P., Xiong, Y., Yue, J., Ihira, K., Konno, Y., et al. (2019). MicroRNA-361: a multifaceted player regulating tumor aggressiveness and tumor microenvironment formation. Cancers (Basel) 11:1130. doi: 10.3390/ cancers 11081130

Xu, S., Lei, S. L., Liu, K. J., Yi, S. G., Yang, Z. L., and Yao, H. L. (2020). circSFMBT1 promotes pancreatic cancer growth and metastasis via targeting miR-3305p/PAK1 axis. Cancer Gene Ther. 28, 234-249. doi: 10.1038/s41417-020-00 215-2.

Zhang, J., and Ma, L. (2012). MicroRNA control of epithelial-mesenchymal transition and metastasis. Cancer Metastasis Rev. 31, 653-662. doi: 10.1007/ s10555-012-9368-6

Zhang, J., Xiao, C., Feng, Z., Gong, Y., Sun, B., Li, Z., et al. (2020). SOX4 promotes the growth and metastasis of breast cancer. Cancer Cell Int. 20:468. doi: 10.1186/ s12935-020-01568-2

Conflict of Interest: The authors declare that the research was conducted in the absence of any commercial or financial relationships that could be construed as a potential conflict of interest.

Copyright (c) 2021 Zhang, Zhu, Ma and Ai. This is an open-access article distributed under the terms of the Creative Commons Attribution License (CC BY). The use, distribution or reproduction in other forums is permitted, provided the original author(s) and the copyright owner(s) are credited and that the original publication in this journal is cited, in accordance with accepted academic practice. No use, distribution or reproduction is permitted which does not comply with these terms. 